\title{
Managing Adrenal Tumors in Children: Tricks and Tips
}

\author{
Roma Pradhan
}

\begin{abstract}
Adrenal tumors are extremely rare in childhood, except in Brazil, so that their clinical manifestations remain poorly known by pediatricians. In children, the most common manifestation of adrenal tumors is the appearance of pubic hair and other signs of virilization. Isolated androgen secretion is present in $40 \%$ of children's tumors vs $9 \%$ in adults. These tumors also have no reliable set of criteria for malignancy. Therefore, all suspected adrenal tumors must be managed as adrenocortical carcinomas (ACCs) until the final diagnosis is made. All children should be treated in endocrine oncologic centers with experienced endocrine surgeons in a highly interdisciplinary setting.
\end{abstract}

Keywords: Adrenal tumor, Adrenocortical carcinoma, Pediatric.

How to cite this article: Pradhan R. Managing Adrenal Tumors in Children: Tricks and Tips. World J Endoc Surg 2018;10(2):145-146.

Source of support: Nil

Conflict of interest: None

\section{INTRODUCTION}

Adrenal tumors are extremely rare in childhood except in Brazil ${ }^{1,2}$ so that their clinical manifestations remain poorly known by pediatricians. In adults, adrenal tumors often are revealed by Cushing syndrome, with evidence of glucocorticoid secretion in two-thirds of patients. ${ }^{3,4}$ These tumors are often large at diagnosis and are already metastatic in 22 to $50 \%$ of patients. In children, the most common manifestation of adrenal tumors is the appearance of pubic hair and other signs of virilization (Fig. 1). Isolated androgen secretion is present in $40 \%$ of children's tumors vs $9 \%$ in adults. The high frequency of virilization should further accelerate the diagnosis, provided that pediatricians systematically use ultrasound and computed tomography (CT) scanning to image adrenals in children of both sexes who present with any androgenic manifestation, even as mild as pubic hair. Also, if children suddenly get obese, particularly those with peripheral precocious puberty, they should be screened for ACTs. The laboratory characteristics of ACTs are the discordantly elevated

\footnotetext{
Assistant Professor

Department of Endocrine Surgery, Dr. Ram Manohar Lohia Institute of Medical Sciences, Lucknow, Uttar Pradesh, India

Corresponding Author: Roma Pradhan, Assistant Professor Department of Endocrine Surgery, Dr. Ram Manohar Lohia Institute of Medical Sciences, Lucknow, Uttar Pradesh, India e-mail: drromapradhan@gmail.com
}

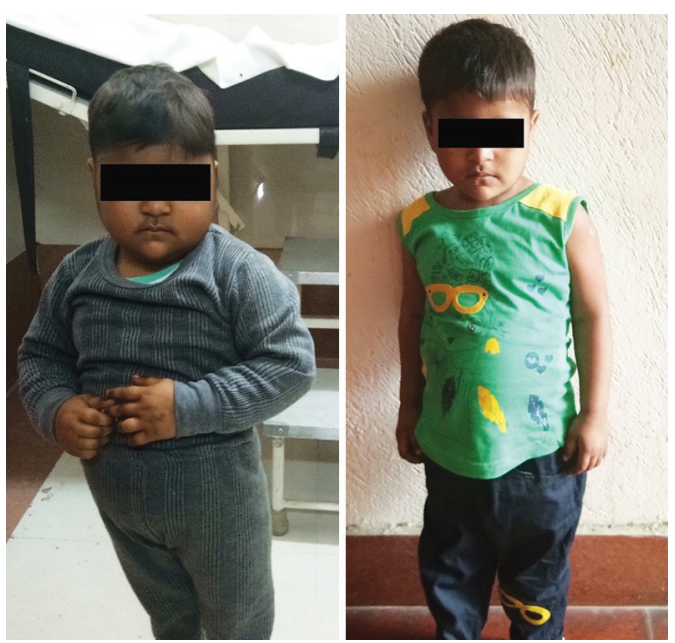

Fig. 1: Preoperative and postoperative clinical image

serum levels of sexual corticosteroids, and disturbance of the normal circadian rhythm of cortisol secretion. Contrast-enhanced CT is the imaging of choice. Classical imaging findings of childhood ACTs consist of a large, well-defined suprarenal tumor containing calcifications with a thin capsule and central necrosis or hemorrhage. But unfortunately, the characteristics of imaging of different adrenal tumors are very similar. It is hard to make a correct diagnosis, especially to determine the malignant nature by imaging alone. Pediatric ACT is relatively small at diagnosis, and metastases are observed in only $13 \%$. These tumors also have no reliable set of criteria for malignancy, ${ }^{3,4}$ and their evolution remains largely unpredictable. In children, the most common manifestation of adrenal tumors is the appearance of pubic hair and other signs of virilization (Fig. 1). Isolated androgen secretion is present in $40 \%$ of children's tumors vs $9 \%$ in adults. The high frequency of virilization should further accelerate the diagnosis. ${ }^{5-7}$ Some pointers for malignancy in pediatric ACT include an ACT with large size in imaging (especially the one with diameter more than $10 \mathrm{~cm}$ ) and/ or extremely high lactate dehydrogenase (LDH) level (especially when LDH is more than two times the upper limit of normal) may predict the malignant nature. This observation is particularly important for the preoperative management of ACTs in children. Therefore, all suspected adrenal tumors must be managed as ACCs until the final diagnosis is made. As almost all ACCs in children could be diagnosed clinically, the need for biopsy in the diagnostic follow-up has to be discussed critically. Biopsies can delay the preoperative diagnosis, providing especially in large 


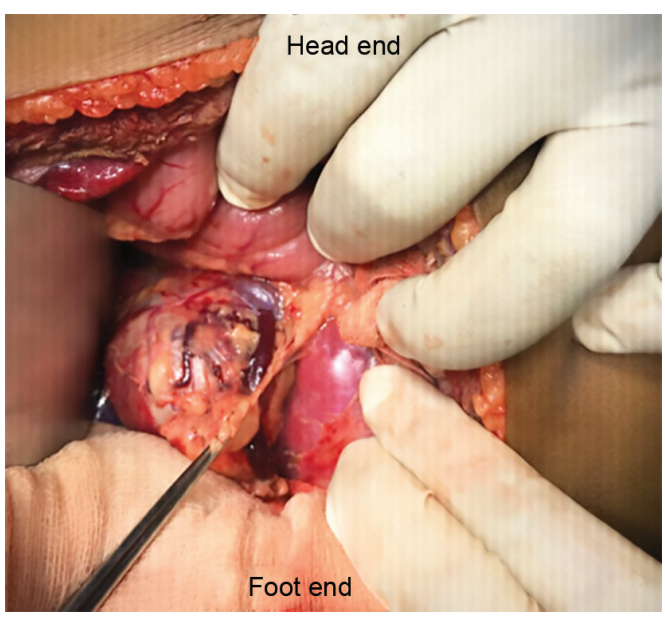

Fig. 2: Intraoperative image

tumors often no reliable and representative histological information, but can also deteriorate the prognosis due to tumor spillage. ${ }^{6}$ In one study, preoperative tumor biopsy was associated with a reduction of the overall survival rate from $69 \%$ in patients who did not undergo biopsy to $42 \%$ in patients who did undergo biopsy. To date, ACCs are only curable by complete resection of the tumor. Therefore, all efforts must be made to achieve R0 resection of the primary tumor. The ACC vulnerability, tumor size, and invasion of surrounding tissues are negative predictive factors and determine the success of primary tumor resection. The vulnerability of the ACC was found to be responsible for the high rate of operative tumor ruptures $(19 \%)$ and is associated with poor outcome. Because of tumor friability, rupture of the capsule with resultant tumor spillage is frequent (approximately $20 \%$ of initial resections and $43 \%$ of resections after recurrence). Thus, a special challenge for the endocrine surgeon, which is crucial for the patient, is the complete resection of the primary tumor without tumor spillage (Figs 2 and 3). The trick is to stay away from the capsule and not to give pressure on the tumor. There is lack of reliable histopathological criteria for diagnosis of malignancy in childhood ACT. Weiss criteria do not accurately predict clinical outcome and are not entirely applicable to pediatric ACC. A modified scoring system, also known as Wieneke criteria, has been since used and has been validated in many studies. TP53 mutation has been implicated in ACT development and progression. Currently, TP53 mutation screening is not applicable as a worldwide diagnostic criterion of pediatric ACT malignancy. Nevertheless, the strong correlation between TP53 mutations and pediatric ACT merits some consideration for genetic testing of TP53 status in specific settings. Efficacy of adjuvant treatment with mitotane in children is doubtful because of significant toxicity. ACTs are generally considered to be radio resistant. Furthermore, because many children with ACT carry germ line TP53 mutations that predispose to cancer, radiation may

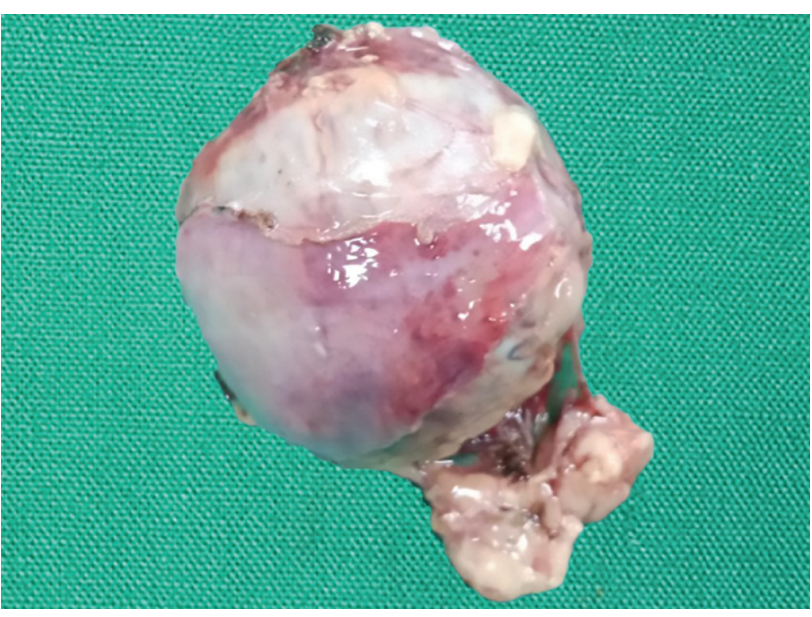

Fig. 3: Specimen

increase the incidence of secondary tumors. Preoperative and perioperative management is important for long-term outcomes of children with ACC. A special challenge for the endocrine surgeon, which is crucial for the patient, is the complete resection of the primary tumor without tumor spillage. Laparoscopic tumor resection in childhood is not recommended due to the risk of tumor rupture. Precise imaging of the tumor, including accurate tumor size, local and vascular invasion pattern, and lymph node involvement, by ultrasound $\mathrm{CT}$ and magnetic resonance imaging techniques, as well as steroid hormone measurements in serum and urine are obligate preoperative diagnostic procedures. All children should be treated in endocrine oncologic centers with experienced endocrine surgeons in a highly interdisciplinary setting.

\section{REFERENCES}

1. Sabbaga CC, Avilla SG, Schulz C, Garbers JC, Blucher D. Adrenocortical carcinoma in children: clinical aspects and prognosis. J Pediatr Surg 1993 Jun;28(6):841-843.

2. Sandrini R, Ribeiro RC, DeLacerda L. Childhood adrenocortical tumors. J Clin Endocrinol 1997 Jul;82(7):2027-2031.

3. Icard P, Chapuis Y, Andreassian B, Bernard A, Proye C. Adrenocortical carcinoma in surgically treated patients: a retrospective study on 156 cases by the French Association of Endocrine Surgery. Surgery 1992 Dec;112(6):972-980.

4. Luton JP, Cerdas B, Billaud L, Thomas G, Guilhaume B, Bertagna X, Laudat MH, Louvel A, Chapuis Y, Blondeau P, et al. Adrenocortical carcinoma: clinical features, prognostic factors and therapeutic results in 105 patients from a single center (1963-1987). N Engl J Med 1990 Apr;322(17):1195-1201.

5. Papotti M, Libé R, Duregon E, Volante M, Bertherat J, Tissier F. The weiss score and beyond-histopathology for adrenocortical carcinoma. Horm Cancer 2011 Dec;2(6):333-340.

6. Rodriguez-Galindo C, Figueiredo BC,Zambetti GP, Ribeiro RC. Biology, clinical characteristics, and management of adrenocortical tumors in children. Pediatr Blood Cancer 2005 Sep;45(3): 265-273.

7. Wieneke JA, Thompson LD, Heffess CS. Adrenal cortical neoplasms in the pediatric population: a clinicopathologic and immunophenotypic analysis of 83 patients. Am J Surg Pathol 2003 Jul;27(7):867-881. 